\title{
Vom Demografiemanagement zur systematischen Personalarbeit ${ }^{1}$
}

\author{
G. Richter, K. Kubn und A.-L. Koch
}

Einleitung: Die politische Ausgangslage um das Jahr 2000

Eine wesentliche Orientierung von Wirtschaft und Politik, aber auch vieler Erwerbstätiger ab Mitte der 90er-Jahre, war auf das vorzeitige Ausscheiden aus dem Erwerbsleben gerichtet. Für Wirtschaft und Politik waren ein sozialverträglicher Personalabbau, die Entlastung des Arbeitsmarktes und Bereitstellung von Arbeitsplätzen für Jüngere (z.B. durch Altersteilzeit vgl. Stück 2003) wichtige Argumente, für viele Beschäftigte stand die Beendigung eines belastenden und anstrengenden Erwerbslebens im Vordergrund. In Kauf genommen wurde der Verlust wertvollen Erfahrungswissens. Sehr schnell wurde jedoch deutlich, dass ein Umdenken in den Politikoptionen nötig ist (siehe dazu den Beitrag von Overhage in diesem Band).

\section{Impulse aus der europäischen Diskussion}

Seit den 90er-Jahren sind vor allem von Skandinavien wichtige Impulse ausgegangen, wenn es um die Forschung zu und die Schaffung von gesunden Arbeitsbedingungen geht. Insbesondere Forschungsarbeiten der finnischen Arbeitsschutzagentur FIOH und ihres Mitarbeiters Juhani Illmarinen, die zur Entwicklung und Validierung eines Fragebogens zur Messung der Arbeitsfähigkeit (Workablity Index, WAI) geführt haben, sind zu nennen. In Deutschland hat die Bundesanstalt für Arbeitsschutz und Arbeitsmedizin (BAuA) 2001 eine Übersetzung dieses Arbeitsfähigkeitsindexes veröffentlicht, dem 2007 ein Leitfaden zur Anwendung zur Seite gestellt wurde (Hasselhorn \& Freude 2007). „Der WAI ermittelt anhand eines Fragenkatalogs einen Punktwert. Dieser zeigt an, inwieweit eine Person hinsichtlich ihrer persönlichen Voraussetzungen und der Arbeitsbedingungen in der Lage ist, aktuell und künftig ihre Arbeit zu verrichten" (vgl. Tempel \& Ilmarinen 2013). Es handelt sich dabei ausdrücklich nicht um eine Maßzahl, die die Gesundheit oder die Leistungsfähigkeit einer bzw. eines Be- 
schäftigten abbildet. Der WAI-Wert gibt vielmehr Auskunft darüber, inwieweit die individuellen Fähigkeiten und Fertigkeiten einer Person mit den gestellten Anforderungen der Arbeit übereinstimmen. Mit anderen Worten: „Beim WAI steht das Potenzial eines Menschen im Mittelpunkt, zu einem bestimmten Zeitpunkt eine bestimmte Arbeitsanforderung zu erfüllen bzw. zu bewältigen“ (INQA 2018).

Juhani Ilmarinen hat mit dem „Haus der Arbeitsfähigkeit“ darüber hinaus ein Modell entwickelt, wie die Arbeitsfähigkeit betrieblich gemanagt und erhalten werden kann. Arbeitsfähigkeit ist die Übereinstimmung zwischen dem, was ein Betrieb dauerhaft verlangt und dem, was eine Person leisten kann und will. Die Faktoren, die diese Übereinstimmung beeinflussen, werden im Modell „Haus der Arbeitsfähigkeit“ zusammengefasst. Es besteht aus den miteinander verknüpften Bereichen Gesundheit, Kompetenz, Werte und Arbeit. Es geht um die Passung zwischen Arbeitenden und Arbeit. Eine gute Arbeitsfähigkeit bedeutet, dass die Menschen mit den ihnen zur Verfügung stehenden Ressourcen die gewünschte Arbeit gut leisten können. Dieses Modell hat dazu geführt, Gesundheitsmanagementsysteme stärker auf ein verlängertes Erwerbsleben auszurichten. Es ist von Sozialversicherungen und Sozialpartnern in vielen Beratungsprojekten aufgegriffen worden und dient als Grundlage und Leitschnur für die Entwicklung gesunder Arbeitsbedingungen und -kulturen.

Die European Foundation for the Improvement of Living and Working Conditions in Dublin hat Ende der 90er-Jahre (Walker 1997) und zu Beginn der 2000er-Jahre (Naegele \& Walker 2006) einen Leitfaden für ein gutes Altersmanagement ${ }^{2}$ entwickelt. Für Naegele und Walker muss Altersmanagement auf Unternehmensebene erfolgen, es bedarf aber der Begleitung durch rechtliche oder politische Maßnahmen für die alternde Erwerbsbevölkerung. Betriebliche und gesellschaftspolitische Maßnahmen werden als sich gegenseitig verstärkend angesehen. Betriebliches Altersmanagement umfasst danach die folgenden acht Dimensionen: 
- Personalbeschaffung

- Lernen, Ausbildung und lebenslanges Lernen

- berufliche Entwicklung

- flexible Arbeitszeitgestaltung

- Gesundheitsschutz, Gesundheitsförderung und Arbeitsplatzgestaltung

- Personalumsetzung

- Erwerbsaustritt und Übergang in den Ruhestand

- ganzheitliche Ansätze

Die Herausforderung besteht darin, die Dimensionen dieses Konzepts in ein integriertes Personalmanagement zu bringen und mit der betrieblichen Arbeits- und Leistungspolitik zu verbinden. Die Demografieperspektive eröffnet den Blick über den Betrieb hinaus und bezieht den lokalen Arbeitsmarkt und das Bildungssystem mit ein. Die Alternsperspektive dynamisiert den Blick auf das Personalmanagement und sensibilisiert für langsame Veränderungsprozesse, Erwerbsverläufe, Lebensphasen und Generationenbeziehungen. Dadurch werden Perspektiven für präventive Strategien sichtbar. Allerdings steigen die Erwartungen an das Personalmanagement und die Komplexität des Aufgabenfeldes nimmt zu.

\section{Nationale übergesetzliche Aktivitäten des BMAS}

Durch zahlreiche Initiativen von Bundes- und Landesregierungen sind Strategien, Instrumente, Vorgehensweisen und Netzwerke etc. entwickelt worden, um den demografischen Wandel bewältigen zu können. Mit der Initiative Neue Qualität der Arbeit (INQA) hat das BMAS vor fast 20 Jahren einen europäischen Impuls (die Lissabon Strategie) aufgegriffen und ein Netzwerk initiiert, in dem zentrale Gestaltungakteure der Arbeitswelt wie die Sozialpartner, die Sozialversicherungsträger, Wirtschaftsverbände, Kammern, Stiftungen und betriebliche Akteure miteinander für eine neue Qualität der Arbeit einsetzen (s. dazu auch den Beitrag von Overhage in diesem Band). „INQA zielt auf die Gestaltung von modernen, an menschlichen Fähigkeiten und Bedürfnissen ausgerichteten Arbeitsbedingungen und auf die Förderung und den Erhalt von Gesundheit und Beschäftigungsfähigkeit. Das Leitmotiv der Zusammenarbeit heißt: „Gemeinsam handeln, jeder in seiner Verantwortung." Mit der Adressierung der Arbeitsqualität knüpft INQA an Forschungen und Modellprojekte der „Hu- 
manisierung der Arbeitswelt“ an. Die Initiative wirkt bis heute auf verschiedenen Ebenen. Dazu zählen

- die Bereitstellung von Informationen und praxisorientierten Handlungshilfen,

- die Unterstützung von Unternehmen bei der Bilanzierung und Weiterentwicklung ihrer Aktivitäten für eine zukunftsgerechte Personalpolitik,

- die zielgerichtete Förderung ausgewählter Forschungs- und Entwicklungsprojekte mit hohem Innovations- und Transferpotenzial sowie

- die Zusammenarbeit mit regionalen und themenspezifischen Partnernetzwerken und Unterstützern.

Besondere Bedeutung kommt in diesem Kontext dem Demographie Netzwerk (ddn) zu, das unter dem Dach von INQA betriebliche Beteiligte in einen Kommunikationszusammenhang brachte, um gemeinsame Lösungen im übergreifenden Interesse zu erarbeiten. Die Mitglieder des Unternehmensnetzwerkes ddn teilen gemeinsame Werte und sind von der Erfolgsdynamik einer mitarbeiterorientierten und generationengerechten Unternehmenskultur überzeugt. Mit den „zehn goldenen Regeln“ haben die Unternehmensvertreterinnen und -vertreter dieses Netzwerkes eine wichtige Zielorientierung für ein betriebliches Demografiemanagement vorgegeben (siehe dazu den Beitrag von Fassio, Schmeink und Zeckra in diesem Band).

Im Rahmen von INQA hat der Thematische Initiativkreis „30, 40, 50 plus - Älter werden in Beschäftigung" unter Beteiligung von Vertreterinnen und Vertretern beider Sozialpartner, aus Wissenschaft, Sozialversicherungen, Unternehmen, Arbeits- und Gesundheitsschutz sowie Beratung programmatische Konzepte erarbeitet. Das erste INQA Memorandum „Demografischer Wandel und Beschäftigung" aus dem Jahre 2004 nahm hauptsächlich das Altern und das Alter als solches in den Blick. So zählte das Memorandum Handlungsstrategien und -beispiele für die gelungene Integration älterer Arbeitnehmerinnen und Arbeitnehmer auf und fokussierte sich dabei auf die demografietaugliche Personalarbeit. Damit wurde die Aufmerksamkeit auf ein Themenfeld gelenkt, das damals in der Arbeitswelt kaum und wenn meist als Defizit betrachtet worden ist. Dem setzt das Memorandum einen differenzierten und auf die Potenziale des Alterns gerichteten Blick entgegen. Das zweite INQA Memorandum „Mit Prävention die Zukunft gewinnen - Strategien für eine demografiefeste Arbeitswelt" aus dem Jahr 2010 hat die Perspektive erweitert. Nun wird die gesamte Erwerbsbiographie in den Blick genommen und dadurch können vermehrt präventive Strategien begründet werden. Mit dem Blick auf 
die Jüngeren geraten auch Prozesse des generationenübergreifenden $\mathrm{Zu}$ sammenarbeitens in das Blickfeld. Das dritte Memorandum „Kompetenz gewinnt" aus dem Jahr 2016 betrachtet den Aufbau und die Entwicklung beruflicher Kompetenzen als zentralen Hebel zum Erhalt der Arbeitsfähigkeit. Damit richtet sich die Aufmerksamkeit auf das berufsvorbereitende, -bezogene und arbeitsintegrierte Lernen. Chancen und Herausforderungen werden nicht nur für unterschiedliche Alters-, sondern auch für Beschäftigtengruppen thematisiert.

Die Zusammenschau der drei Memoranden zeigt eine Weiterentwicklung der Perspektive. Neben Altersgruppen werden im letzten Memorandum weitere Intersektionen, z.B. das Geschlecht, thematisiert. Intersektionen meint dabei die Überschneidung verschiedener Persönlichkeitsmerkmale wie Alter und Geschlecht und analysiert damit verbundene Diskriminierung. Der Begriff wurde Mitte des letzten Jahrhunderts von der schwarzen feministischen Bewegung („black feminism movement“) in den USA geprägt und thematisiert die Überschneidung verschiedener gesellschaftlicher Kategorien, wie z.B. Geschlecht, Hautfarbe oder Herkunft, und beschreibt darauffolgende Subordination (Walgenbach 2012). Die Perspektive der Intersektionalität ermöglicht eine Sensibilisierung für die spezifischen Probleme mehrfach subordinierter Gruppen. Die intersektionale Perspektive kann weitergedacht werden, denn Beschäftige mittleren Alters und jüngere Beschäftigte stehen vor anderen Herausforderungen als ältere Arbeitnehmerinnen und Arbeitnehmer oder solche, die z.B. durch längere Arbeitsausfälle nicht in ihrem Beruf tätig waren und denen es deshalb an typischen Berufserfahrungen fehlt. Die Intersektionen sind scheinbar zahllos, aber eins ist ihnen gemein: Sie fordern einen individualisierten Umgang mit den spezifischen Herausforderungen, die sich aus der Lebensund Erwerbsbiografie Einzelner ergeben. Die Perspektive auf die gesamte Erwerbsbiografie und auf Intersektionen in den letzten beiden INQA Memoranden hilft, die vielschichtigen Wandlungsprozesse der modernen, globalisierten Arbeitswelt zu erfassen.

\section{Entwicklung und Förderung des betrieblichen Demografiemanagements}

Betriebliches Demografiemanagement ist eine ,Erfindung' der 2000er-Jahre. In der Dokumentation der Tagung Arbeit und Altern des Jahres 1999 (von Rothkirch, 2000) kommt der Begriff nicht vor. Das Alter von Arbeitnehmerinnen und Arbeitnehmern als solches ist zunächst kein Problem, das sich einer Berufsausübung entgegenstellen oder diese erschweren würde. Zum Problem wird das Altern im Berufsleben erst dann, wenn Be- 
schäftigte auf lange Sicht in belastungsintensiven Tätigkeiten verbleiben und wenn sich eine dort geforderte spezifische Belastbarkeit so weit verbraucht, dass das individuelle Leistungsvermögen den Anforderungen am Arbeitsplatz immer weniger genügt. Das gilt nicht nur für Berufe, in denen schwerste körperliche Arbeit gefordert wird, sondern ebenso für solche, in denen bestimmte psychische Belastungen überwiegen. Zeigen sich Alter und nachlassende Leistungsfähigkeit gleichzeitig bei Beschäftigten, die gemäß ihrem kalendarischen Alter von 40, 50 oder 60 Jahren eigentlich „mitten im Leben stehen", so hängt dies mit den kumulierten Belastungswirkungen aus der bisherigen Berufstätigkeit zusammen. Dennoch wird die Tatsache, dass ältere Arbeitnehmerinnen und Arbeitnehmer aus belastungsintensiven Arbeitsbereichen oftmals weniger leistungsfähig sind, immer wieder der "Natur" oder dem Alter als solchem zugeschrieben. Die verminderte Leistungsfähigkeit Älterer wird zum Stereotyp, zur pauschalen, nicht überprüften Aussage. Dadurch wird aber der Entstehungszusammenhang ebenso verdeckt wie individuelle Unterschiede.

Ein krankheitsbedingter Verlust der Arbeitsfähigkeit steht nach wie vor häufig in engem Zusammenhang mit der Tätigkeit bzw. den Bedingungen, unter denen diese verrichtet wird oder in der Vergangenheit verrichtet wurde. Der Gesundheitszustand eines Beschäftigten ist also meist nicht primär durch das kalendarische Alter determiniert, sondern oftmals Ergebnis der Arbeitsbedingungen der Vergangenheit. Es gibt viele Belege, denen zufolge eine nachlassende körperliche Leistungsfähigkeit nicht altersbedingt, sondern durch eine Vielzahl exogener Fakten zu erklären ist. Dies unterstreicht die große Unterschiedlichkeit der körperlichen Leistungsfähigkeit Gleichaltriger. Somit ist der ältere Mensch auch heute noch „eine gemachte Problemgruppe".

\section{Anforderungen an systematisches Personalmanagement}

Der Rückblick auf die Konzepte und Initiativen der vergangenen 20 Jahre lässt sich in sechs Themenfeldern zusammenfassen.

1. Von einer flächendeckenden Verbreitung des Demografiemanagements in deutschen Unternehmen und Verwaltungen kann im Jahr 2020 keine Rede sein. Dieser Ansatz hat sich nicht durchgesetzt. Das bedeutet jedoch nicht, dass der demografische Wandel bei Personalverantwortlichen keine Rolle spielt. Bei der letzten Welle des ifaa Trendbarormeters ${ }^{3}$ vor Ausbruch der Corona-Pandemie, im Herbst 2019, besetzten folgende Themen die Top 5: Prozessorganisation, Fachkräftesicherung, kontinuierliche Verbesserung (KVP), Arbeitszeitflexibilität, gesetzl. Arbeits- und Ge- 
sundheitsschutz. Von diesen fünf relevanten Themen adressieren mit Fachkräftesicherung, Arbeitszeitflexibilität und gesetzlichem Arbeits- und Gesundheitsschutz drei Themen wichtige Aspekte der betrieblichen Demografiepolitik.

Der Ansatz des betrieblichen Demografiemanagements als eigenständigem Handlungsfeld mit exklusiver Zuständigkeit von speziellen Expertinnen oder Experten hat sich also nicht durchgesetzt (siehe dazu auch den Beitrag von Reindl in diesem Band). Dies hängt sicherlich auch damit zusammen, dass es keine generelle Betroffenheit der Betriebe und Verwaltungen vom demografischen Wandel gibt. Vielmehr spielen Faktoren wie Region und räumliche Lage, Branche, Anforderungsprofil, Wettbewerbssituation, Unternehmensgeschichte, aber auch der Ruf des Unternehmens eine große Rolle dafür, von welchen Aspekten des demografischen Wandels eine Organisation in welchem Ausmaß betroffen ist. Kurz: Nicht alle Unternehmen und Verwaltungen brauchen Demografiemanagement. Aber alle Betriebe und Verwaltungen brauchen eine qualifizierte Arbeitsgestaltung und eine systematische Personalarbeit auf der Grundlage des gesetzlichen Arbeits- und Gesundheitsschutzes.

Ein systematisches betriebliches Personalmanagement integriert Arbeits- und Gesundheitsschutz, betriebliche Gesundheitsförderung und das Altersmanagement und zielt darüber hinaus auf die Führung, die Unternehmenskultur, das Betriebsklima, die soziale Kompetenz, auf die Arbeitsbedingungen und das Gesundheitsverhalten. Es stützt sich auf die regelmäßige Anwendung von validierten Instrumenten wie Altersstruktur-, Qualifikationsbedarfs- und Kompetenzanalysen sowie die Gefährdungsbeurteilung. Dadurch ist es mit der Arbeitsgestaltung und der Organisations- und Personalentwicklung verbunden. Es betrachtet die betriebliche Personalarbeit im Kontext des regionalen Arbeitsmarktes. Dieses Personalmanagement ist mit der Unternehmensstrategie verknüpft und kann deshalb strategische Personalarbeit betreiben. Der Erhalt der Arbeits- und Beschäftigungsfähigkeit ist ein zentrales Ziel und Erfolgsindikator der systematischen Personalarbeit.

2. Um den demografischen Wandel als Chance nutzen und gestalten zu können ist ein erweitertes Präventionsverständnis zu entwickeln und zu praktizieren. Dieses erweiterte Präventionsverständnis ist vor allem durch zwei Merkmale gekennzeichnet: Es nimmt eine langfristige Perspektive ein und integriert Maßnahmen in den Feldern Arbeits- und Laufbahngestaltung, Gesundheit und Qualifizierung. Dieser Ansatz geht über die klassische betriebliche Gesundheitsförderung hinaus und integriert Ansätze u.a. des lebensbegleitenden Lernens mit der Zielsetzung der Sicherung der Be- 
schäftigungsfähigkeit. Zum Beispiel wird das Wechseln von Tätigkeiten im Lebensverlauf - und somit auch der Wechsel von Belastungen - einfacher.

Die Aufgabe von Sozialversicherungsträgern, Sozialpartnern und der Politik muss sein, Rahmenbedingungen zu schaffen, die ein solches Präventionsverständnis und die dazu nötige Flexibilität unterstützen und absichern.

3. Entscheidend für die Zukunftsfähigkeit der systematischen Personalpolitik ist die strategische Personalplanung: Diese beschreibt die Planung quantitativer und qualitativer Mitarbeiterkapazitäten im Rahmen der strategischen Unternehmensplanung. Sie soll sicherstellen, dass kurz-, mittelund langfristig notwendige Fähigkeitsprofile von Mitarbeiterinnen und Mitarbeitern in Qualität und Quantität zum richtigen Zeitpunkt, am richtigen Ort und zu den richtigen Kosten zur Verwirklichung der Unternehmensziele zur Verfügung stehen (Schwuchow/Gutmann 2015). Dabei sollten folgende Faktoren berücksichtigt werden:

- Der Schwerpunkt liegt auf der Vermeidung altersbedingter Probleme wie der Dequalifizierung älterer Arbeitnehmerinnen und Arbeitnehmer oder arbeitsbedingter gesundheitlicher Probleme und nicht so sehr auf einer nachträglichen Problemlösung.

- Im Mittelpunkt sollten nicht nur die älteren Arbeitnehmerinnen und Arbeitnehmer, sondern das gesamte Arbeitsleben und alle Altersgruppen stehen.

- Wichtig ist ein integrierter Ansatz, der sämtliche Dimensionen und Akteure umfasst, die zu einer effektiven Umsetzung des Altersmanagements beitragen.

- Umdenken innerhalb des Unternehmens und der Gesellschaft und Sensibilisierung für die Notwendigkeit der Altersvielfalt.

- Kurzfristig sind Fort- und Weiterbildungsmaßnahmen für ältere Arbeitnehmerinnen und Arbeitnehmer vorzusehen, die keine Fachausbildung absolvieren konnten oder bei denen aufgrund der Berufstätigkeit gesundheitliche Probleme aufgetreten sind.

- Durchführung regelmäßiger strategischer Evaluierungen von Altersmanagement-Strategien und -Initiativen zur Bewertung ihrer Wirksamkeit.

Die Verwirklichung dieser Strategie würde dafür sorgen, dass Unternehmen ihre Investitionen in die vorhandene Belegschaft als strategische Investitionen betrachten und maximieren.

4. Dies gilt besonders für die große Zahl kleiner Unternehmungen. Eine Bilanzierung der vergangenen 20 Jahre betrieblichen Personalmanagements aus der Demografieperspektive kann nicht übersehen, dass es in vie- 
len kleinen Betrieben an qualifizierter Arbeitsgestaltung und systematischer Personalarbeit auf Grundlage des gesetzlichen Arbeits- und Gesundheitsschutzes mangelt. Insbesondere Betriebe ohne eigene Kompetenzen in diesen beiden Feldern rücken damit in den Blickpunkt. Punktuell bringen Beraterinnen und Berater, etwa von Kammern oder Sozialversicherungen, die fehlende Expertise in die Betriebe. Das Bundesarbeitsministerium und einzelne Landesregierungen haben ebenfalls Förderprogramme zur Unterstützung von Kleinbetrieben aufgelegt und Zusammenschlüsse von Beraterinnen und Beratern wie die Offensive Mittelstand gefördert. Gleichwohl bleiben diese Angebote projekthaft finanziert, in der Ausrichtung kurzatmig und vor allem der Förderlogik von Programmen verpflichtet, nicht aber den Anforderungen von Betrieben und Beschäftigten. Heute wie vor 20 Jahren fehlt eine abgestimmte und dem Erhalt der Arbeitsfähigkeit verpflichtete Unterstützungsinfrastruktur für Kleinbetriebe. Sozialversicherungen, Kammern, kommunale Wirtschaftsförderung, Gewerbeaufsicht, Arbeitsagenturen und Job-Center bearbeiten Teilaspekte und folgen jeweils unterschiedlichen institutionellen Logiken.

5. Damit steht die flächendeckende Förderung einer systematischen Personalarbeit auch in Kleinbetrieben auf der Agenda. Diese systematische Personalarbeit integriert die Handlungsfelder des Demografiemanagements. Vor allem ist sie durch eine Orientierung an der Lebenslaufperspektive gekennzeichnet und damit stärker individuell ausgerichtet. Die Lebenslaufperspektive stärkt auch die dynamische Sicht und ein Bewusstsein für die Notwendigkeit von Veränderung. Zugleich wohnt der Lebenslaufperspektive eine Langfristorientierung inne, die zur Notwendigkeit führt, Unternehmens- und Personalperspektive strategisch zu verknüpfen. Damit dieses Bild angesichts der durch zunehmende Unsicherheiten geprägten Märkte (Digitalisierung, Corona, Brexit) kein Wunschbild bleibt ist eine begleitende Unterstützungsinfrastruktur erforderlich.

Mit der Verabschiedung des Präventionsgesetzes ist der Stellenwert präventiver Strategien in Arbeits- und Lebenswelt zumindest auf dem Papier aufgewertet worden. Ob diese Aufwertung allerdings reicht, das oben bereits deutlich gewordene Defizit an Abstimmung zwischen den verschiedenen Akteuren zu überwinden muss bezweifelt werden. Vor allem, weil bereits 2000 auf diese Schwäche im System der gegliederten Verantwortung hingewiesen worden ist: „Letztendlich brauchen wir eine bessere Passung der unterschiedlichen Politiken: im Bereich der Arbeitsmarktpolitik, der Gesundheitspolitik, der Rentenpolitik, der Bildungspolitik. All diese Politiken haben eigenständige Instrumentarien entwickelt, die aber nicht ineinandergreifen. Dies läßt sich gerade am Problem der Berufe mit begrenzter Tätigkeitsdauer - die Folge sind gesundheitlicher Verschleiß, Entmuti- 
gung, mangelnde Neuqualifikation - festmachen“ (Kuhn 2000). Neben einer besseren Passung, das ist durch die starke Verbreitung des CoronaVirus in einigen industriellen Schlachthöfen deutlich geworden, braucht es eine Aufwertung des Arbeitsschutzes und eine stärkere Berücksichtigung arbeitswissenschaftlicher Erkenntnisse in allen Betrieben.

Sobald die Generation der Babyboomer in Rente geht, ist mit einem Rückgang des Arbeitskräfteangebots zu rechnen. Die Folgen werden vielfältig sein. Die Nachfrage nach Arbeitskräften wird sich anpassen müssen, um mit dem zunehmenden Personalmangel umzugehen. Vor allem aber gerät das Sozialversicherungssystem in Schieflage. Ein Rentensystem wie das deutsche ist konzeptionell nicht auf eine schrumpfende Erwerbsbevölkerung ausgelegt. Im Jahr 2018 kamen auf 100 Arbeitskräfte im Alter von 20 bis 64 Jahren 42 Menschen im Rentenalter von 65 oder mehr Jahren. Im Jahr 2035 werden es selbst im günstigsten IAB Szenario 57 ältere Menschen auf 100 Arbeitskräfte sein. Unter diesen Umständen kann man sich nicht mehr auf den sogenannten Generationenvertrag verlassen. (IAB Forum 2020). Damit werden Personalmangel und Älterwerden im Betrieb zur politischen Herausforderung für die nächsten Jahre. Um die oben angesprochene Passung der unterschiedlichen Politikbereiche sicherzustellen, sollte auf die Erfolgsmechanismen der letzten 20 Jahre zurückgegriffen werden, nämlich den Erhalt und die politische Förderung der Kommunikationsfähigkeit zwischen den unterschiedlichen Handlungsträgern durch die Einbindung und weitere Förderung von existierenden Netzwerken.

6. Die Konzepte für das betriebliche Demografiemanagement sind durch verschiedene Modernisierungsmerkmale (Van der Loo/van Reijen 1992) gekennzeichnet. Als Modernisierung werden danach Prozesse bezeichnet, die durch Differenzierung, Rationalisierung, Individualisierung und Domestizierung gekennzeichnet sind.

- Betriebliche Demografiepolitik ist ein Prozess der Differenzierung und umfasst nicht nur das Alter, sondern auch Geschlecht und Herkunft. Die Perspektive auf die gesamte Erwerbsbiographie und auf die verschiedenen Intersektionen ermöglicht präventive Strategien für die Wandlungsprozesse der modernen, globalisierten Arbeitswelt.

- Vorurteile und Stereotype, die das Defizitmodell des Alters gekennzeichnet haben, wurden infrage gestellt und auf Grundlage neuer wissenschaftlicher Erkenntnisse überwunden, so dass von einer Rationalisierung gesprochen werden kann. 
- Die Aufmerksamkeit für betriebliche Spezifikationen und interindividuelle Unterschiede ist gestiegen und deshalb kann ein Prozess der Individualisierung festgestellt werden.

- Arbeits- und Gesundheitsschutz, präventive Arbeitsgestaltung und die Anpassung der Arbeit an den Menschen mit dem Ziel der verlängerten Nutzung der Arbeitskraft sind Kernbestandteil betrieblicher Demografiepolitik. Damit sind wichtige Merkmale einer Domestizierung gegeben.

Abschließend lässt sich zusammenfassen: Demografie bedeutet Anerkennung von Unterschieden. Das Thema Demografie war ein Modernisierungsimpuls für die Personalarbeit. Manifest geht es in Rente, latent bleibt es als Modernisierungsgewinn für die Personalarbeit der 2020er-Jahre von hoher, in vielen Betrieben und Verwaltungen sogar zunehmender Relevanz.

\section{Literatur}

Bundesagentur für Arbeit (BA) (2020): Arbeitslosigkeit im Zeitverlauf.

Crenshaw, K. (1995): Race, reform, and retrenchment: Transformation and legitimation in antidiscrimination law. In: Crenshaw, K. et al. (Hrsg.), Critical race theory. The key writings that formed the movement (S.103-126). New York: The New Press.

Das Demographie Netzwerk e.V. (ddn) (2015): Die 10 goldenen Regeln. https://ww w.demographie-netzwerk.de/ddn/10-goldene-regeln/. Abgerufen am 7.7.2020.

Hasselhorn, H. M. \& Freude, G. (2018): Der Work Ability Index - ein Leitfaden. https://www.wainetzwerk.de/uploads/PDF/Der\%20Work\%20Ability\%20Index\% 20-\%20ein\%20Leitfaden.pdf. Abgerufen am 18.8.2020.

Ilmarinen, J. (2000): Die Arbeitsfähigkeit kann mit dem Alter steigen. In: Rothkirch, v. C. (Hrsg.), Altern und Arbeit. Herausforderung für Wirtschaft und Gesellschaft, Beiträge, Diskussionen und Ergebnisse eines Kongresses mit internationaler Beteiligung (S. 88-96). Berlin: Edition Sigma.

Initiative Neue Qualität der Arbeit (inqa) (Hrsg.) (2014): Tarifverträge zur Gestaltung der Qualität der Arbeit. Ein aktueller Überblick über Vereinbarungen zu alternsgerechten und demografiefesten Arbeitsbedingungen. https:/inqa.de/Sha redDocs/downloads/webshop/tarifvertraege-zur-gestaltung-der-qualitaet-der-arbe it?_blob=publicationFile. Abgerufen am 18.8.2020.

Initiative Neue Qualität der Arbeit (inqa) (Hrsg.) (2018): Eine Familie stellt sich vor WAI-basierte Instrumente - Arbeits- und Beschäftigungsfähigkeit wiederherstellen, erhalten und fördern. https://inqa.de/SharedDocs/downloads/webshop/e ine-familie-stellt-sich-vor-wai?_blob=publicationFile. Abgerufen am 9.7.2020. 
Institut für angewandte Arbeitswissenschaften e.V. (Ifaa) (2019): Topthema der Experten: Leistungsentgelt bei kleinen Betrieben im Fokus. In: ifaa. Trendbarometer. Arbeitswelt. Auswertung Herbst 2019. https://www.arbeitswissenschaft.net/f ileadmin/Bilder/Angebote_und_Produkte/Studien/Auswertung_Herbst_2019_3. pdf. Abgerufen am 7.7.2020.

Klinger S. \& Fuchs, J. (2020): Wie sich der demografische Wandel auf den deutschen Arbeitsmarkt auswirkt. https:/www.iab-forum.de/wie-sich-der-demografis che-wandel-auf-den-deutschen-arbeitsmarkt-auswirkt/. Abgerufen am 18.8.2020.

Kuhn, K. (2000): Arbeitsgruppe 5: Berufe mit begrenzter Tätigkeitsdauer. Zusammenfassung. In: Rothkirch, v. C. (Hrsg.), Altern und Arbeit. Herausforderung für Wirtschaft und Gesellschaft, Beiträge, Diskussionen und Ergebnisse eines Kongresses mit internationaler Beteiligung (S. 276-281). Berlin: Edition Sigma.

Maintz, G. (2000): Arbeitsgruppe 1: Wandel der Leistungspotenziale im Prozess des Alterns - Zusammenfassung. In: Rothkirch, v. C. (Hrsg.), Altern und Arbeit. Herausforderung für Wirtschaft und Gesellschaft, Beiträge, Diskussionen und Ergebnisse eines Kongresses mit internationaler Beteiligung (S. 97-101). Berlin: Edition Sigma.

Naegele, G. \& Walker, A. (2006): A Guide to Good Practice in Age Management. European Foundation for the Improvement of Living and Working Conditions. Office for Official Publications of the European Communities.

Schwuchow, K \& Gutmann, J. (2015): Personalentwicklung. Themen, Trends, Best Practices 2016. Freiburg: Haufe-Lexware.

Stück, H. (2003): Gleitend in den Ruhestand. Gesetzliche, tarifliche und tatsächliche Entwicklung der Altersteilzeit. Arbeitnehmerkammer Bremen.

Tempel, J. \& Ilmarinen, J. (2013): Arbeitsleben 2025. Das Haus der Arbeitsfähigkeit im Unternehmen brauen. Giesert, M. (Hrsg.). Hamburg: VSA.

van der Loo, H. \& van Reijen, W. (1992): Modernisierung. Projekt und Paradox. München: Deutscher Taschenbuch-Verlag.

Walgenbach, K. (2012): Intersektionalität - eine Einführung. http://portal-intersekt ionalitaet.de/theoriebildung/ueberblickstexte/walgenbach-einfuehrung/. Abgerufen am 22.7.2020.

Walker, A. (1997): Maßnahmen zur Bekämpfung von Altersbarrieren in der Erwerbstätigkeit: ein europäischer Forschungsbericht. Europäische Stiftung zur Verbesserung der Lebens- und Arbeitsbedingungen. Dublin.

\section{Referenzen}

1 Wir danken Alfons Schröer und Sigrun Mantei für kritische Hinweise.

2 Die Begriffe Demografiemanagement und betriebliches Altern(s)management bzw. Age-Management werden im Weiteren synonym behandelt.

3 Das ifaa-Trendbarometer "Arbeitswelt" gibt Auskunft über die Bedeutung von Themen der Arbeits- und Betriebsorganisation sowie der Arbeitswissenschaft. In der Zeit von Juli 2019 bis Dezember 2019 haben sich 507 Personen aus Wirtschaft, Verbänden und Wissenschaft an der Befragung beteiligt. Die Expertinnen 
und Experten, die ihre Einschätzungen abgegeben haben, kamen mehrheitlich aus der Wirtschaft (60\%). Der Anteil der Expertinnen und Experten aus den Verbänden lag bei $17 \%$. Aus der Wissenschaft haben sich 7\% und aus sonstigen Bereichen 16\% beteiligt. Bei den Teilnehmerinnen und Teilnehmern aus der Wirtschaft wurde die Betriebsgröße der Unternehmen erfasst. Die Rückläufe stammen anteilig zu 23\% aus Unternehmen mit mehr als 500 Beschäftigen, zu 26\% aus Unternehmen mit 100 bis 499 Beschäftigten und zu 11\% aus Unternehmen mit bis zu 99 Beschäftigten. 
